\title{
Magnetic Ordering in Solid Oxygen up to Room Temperature
}

\author{
S. Klotz, ${ }^{1, *}$ Th. Strässle, ${ }^{2}$ A. L. Cornelius, ${ }^{1,3}$ J. Philippe, ${ }^{1}$ and Th. Hansen ${ }^{4}$ \\ ${ }^{1}$ IMPMC, CNRS-UMR 7590, Université Pierre et Marie Curie, 75252 Paris, France \\ ${ }^{2}$ Laboratory for Neutron Scattering, ETH Zurich and Paul Scherrer Institut, 5232 Villigen PSI, Switzerland \\ ${ }^{3}$ Permanent address: Department of Physics, University of Nevada, Las Vegas, Nevada 89154-4002, USA \\ ${ }^{4}$ Institut Laue Langevin, BP 156, F-38042 Grenoble, France \\ (Received 15 January 2010; published 16 March 2010)
}

\begin{abstract}
Oxygen is the only elemental molecule which carries an electronic magnetic moment. As a consequence, the different solid phases encountered on cooling show various degrees of magnetic order, and similar behavior is expected under compression. Here we present neutron diffraction data which reveal the magnetic ordering under high pressure in the $\delta$ ("orange") phase, i.e., in the range 6-8 GPa and $20-240 \mathrm{~K}$. We show that $\delta-\mathrm{O}_{2}$ contains in total three different magnetic structures, all of them being antiferromagnetic and differing in the stacking sequence of $\mathrm{O}_{2}$ sheets along the $c$ axis. This structural diversity can be explained by the quasi-two-dimensional nature of $\delta$ - $\mathrm{O}_{2}$ and the strong orientation dependence of the magnetic exchange interaction between $\mathrm{O}_{2}$ molecules. The results show that $\delta$ - $\mathrm{O}_{2}$ is a room temperature antiferromagnet.
\end{abstract}

DOI: 10.1103/PhysRevLett.104.115501

Numerous experiments carried out within the last four decades have revealed the existence of five solid phases in oxygen in the $0-10 \mathrm{GPa}$ range (Fig. 1), denoted $\gamma, \beta, \alpha, \delta$, and $\epsilon$, which are, respectively, cubic, rhombohedral, monoclinic, orthorhombic, and monoclinic [1,2]. The former three phases exist at ambient pressure which has allowed detailed investigations of the magnetism in these structures: $\gamma-\mathrm{O}_{2}$ is paramagnetic and converts at $44 \mathrm{~K}$ to the magnetically short-range-ordered $\beta$ phase, which itself transforms at $24 \mathrm{~K}$ into fully antiferromagnetically ordered $\alpha$ oxygen. In contrast, $\delta$ and $\epsilon-\mathrm{O}_{2}$ can only be investigated under high pressure, and consequently our knowledge on magnetism in these phases is very sparse. Indirect evidence of magnetic ordering has been derived from optical spectroscopy without revealing the nature of the ordering [3]. The only neutron diffraction measurement carried out so far suggests the absence of magnetism in $\epsilon-\mathrm{O}_{2}$ [4] as well as in $\delta-\mathrm{O}_{2}[5]$ above $\sim 120 \mathrm{~K}$, but claims antiferromagnetic long range order below $\sim 120 \mathrm{~K}$ based on the observation of a single magnetic reflection. These findings appear inconsistent with the spectroscopic data [3], and a comprehensive understanding of magnetic ordering in $\delta-\mathrm{O}_{2}$ is still lacking.

We have investigated magnetism in $\delta$ - $\mathrm{O}_{2}$ using neutron diffraction and a high pressure technique which allows a sample volume of $\sim 70 \mathrm{~mm}^{3}$. The several orders of magnitude increased intensity compared to previous investigations [5] allowed us to visit most of the stability domain in a reasonable amount of time, i.e., from $20 \mathrm{~K}$ up to almost room temperature and pressures up to $8 \mathrm{GPa}$, the phase boundary with $\epsilon-\mathrm{O}_{2}$. It also permitted the use of Rietveld refinement methods to derive both the nuclear and magnetic structures simultaneously, and investigate their mutual interplay.
PACS numbers: $61.50 . \mathrm{Ks}, 64.70 . \mathrm{kt}, 75.25 .-\mathrm{j}, 75.50 . \mathrm{Xx}$

Several polycrystalline samples of $\delta-\mathrm{O}_{2}$ were prepared at $\sim 240 \mathrm{~K}$ by compression of the liquid through phases $\gamma$ and $\beta$ (Fig. 1) which produced reasonably good powders with some preferred orientation as revealed by Rietveld fits. In two loadings data were collected on cooling $(0.2 \mathrm{~K} / \mathrm{min})$ along the $P-T$ paths indicated in Fig. 1 . The nature of the pressure technique does not allow for cooling at strictly constant pressure. Instead, cooling is quasi-isochoric (as confirmed by the diffraction data shown further below), which leads to a small $(\sim 5 \%-10 \%)$ pressure drop due to the thermal contraction of the sample [6]. Neutron powder diffraction patterns (Fig. 2) collected at high temperatures (up to $235 \mathrm{~K}$ ) reveal immediately the

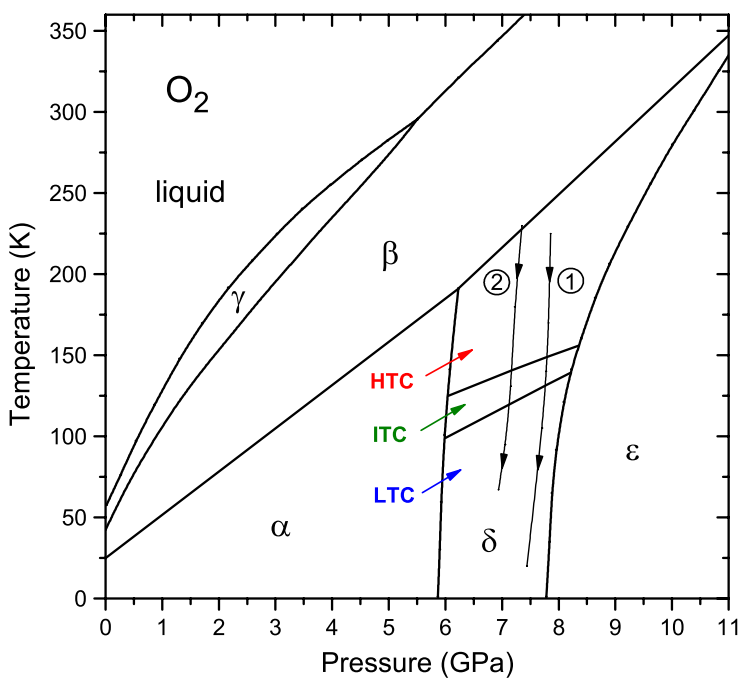

FIG. 1 (color online). Phase diagram of oxygen. The thin vertical lines (1) and (2) indicate trajectories along which diffraction data were collected in two loadings [6]. 


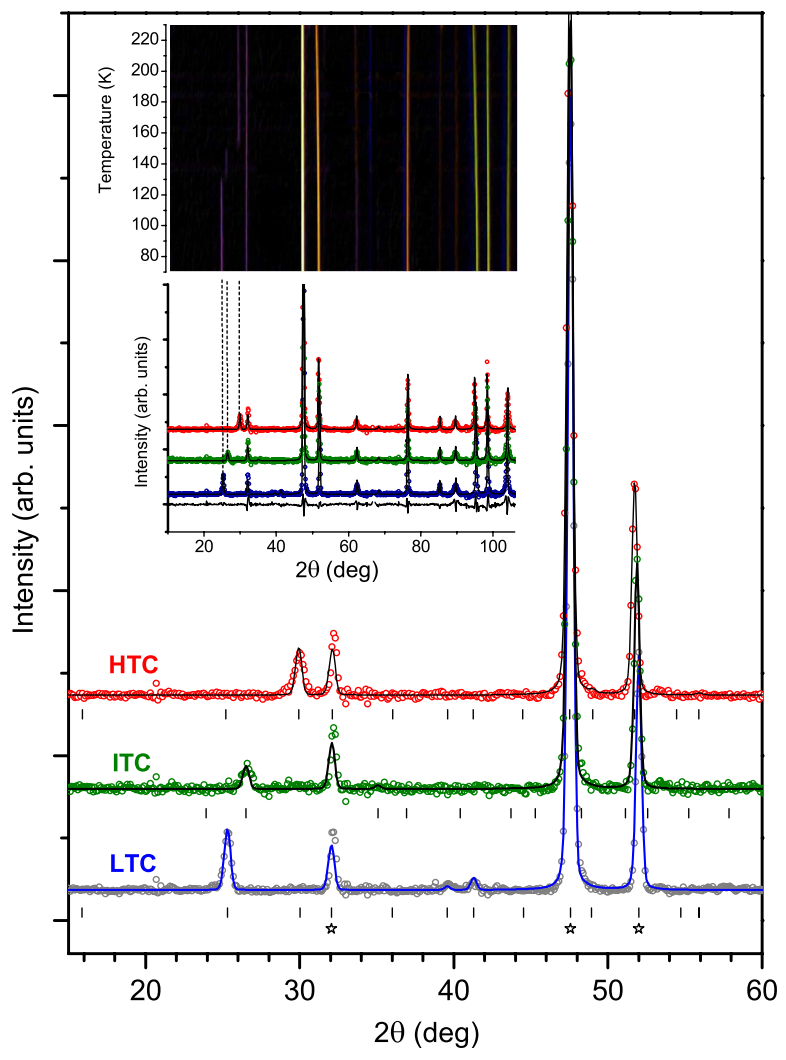

FIG. 2 (color online). Neutron powder diffraction patterns of $\delta$ - $\mathrm{O}_{2}$ in three different magnetic phases LTC (low temperature commensurate), ITC (intermediate temperature commensurate) and HTC (high temperature commensurate) $(\lambda=1.87 \AA)$. The lines through the data (circles) are Rietveld fits to the nuclear and magnetic structure. Tickmarks correspond to positions of magnetic reflections, stars to those of the nuclear (002), (111), (200) Bragg peaks. The inset shows the three patterns over the full $2 \theta$ range, with a difference plot shown underneath, as well as a map of 32 patterns over a temperature range of $160 \mathrm{~K}$. The experiments were carried out at the D20 diffractometer [12] of the Institute Laue Langevin, Grenoble, France. Oxygen (99.998\% purity from Air Liquide) was condensed at $77 \mathrm{~K}$ into a loading clamp and then transferred at $300 \mathrm{~K}$ to a VX5 Paris-Edinburgh cell for data collection. The rest of the experimental setup was identical to the one described in Ref. [13]. The refinements were carried out using FULLPROF [14], refining a minimal set of variables: i.e., a scale factor, background, lattice and profile parameters, the internal oxygen position, the magnetic moment and to some extent anisotropic atomic displacement factors. Magnetic form factors for oxygen were taken from Ref. [9]. The final refinements included a preferred orientation along $\langle 001\rangle$. In loading 1 the (004) reflection was excluded from the fits due to pronounced texture.

presence of magnetic order due to a strong magnetic reflection at $2 \theta=30^{\circ}$. This is, however, not consistent with the reported magnetic structure [5], but corresponds to a spin alignment which resembles the one in $\alpha-\mathrm{O}_{2}$ and which we call here HTC (high temperature commensurate) $\delta$-phase. Upon cooling, this magnetic reflection disappears at $149 \mathrm{~K}$ and another magnetic peak appears at $2 \theta=$ $26.5^{\circ}$. This again cannot arise from the proposed magnetic order [5], but derives from an antiferromagnetic structure with a doubling of the $c$ axis, as explained further below, subsequently called ITC (intermediate temperature commensurate) magnetic phase. This structure is stable over only $\sim 20 \mathrm{~K}$, and transforms at $132 \mathrm{~K}$ into a magnetic phase with a strong reflection at $2 \theta=25^{\circ}$ which is then consistent with the previously observed [5] structure which we subsequently refer as LTC (low temperature commensurate) phase. We repeated these measurements with a different sample at $0.5 \mathrm{GPa}$ lower pressure (path 2, Fig. 1) and found essentially the same results, except that the transitions were shifted to lower temperatures. Interestingly, none of these transitions have a detectable influence on the nuclear structure; i.e., there is no evidence of a pronounced coupling between the magnetic and nuclear degrees of freedom.

Figure 2 includes results from simultaneous Rietveld refinements of the patterns to the nuclear and magnetic structures. Whereas the former is straight forward, the identification of the magnetic structures using at most three reflections is less obvious. The nuclear structures of phases $\alpha, \beta$ and $\delta$ consist of weakly bonded sheets of $\mathrm{O}_{2}$ molecules [2]. The bulk of experimental and theoretical work on solid $\alpha$ - and $\beta$-oxygen shows conclusively that the magnetic interaction between nearest neighbor molecules within these sheets is antiferromagnetic [2]. Given the very close structural relationship with $\alpha-\mathrm{O}_{2}$, it is unquestionable that $\delta-\mathrm{O}_{2}$ adopts a similar in-plane spin arrangement. The problem of the magnetic structure of the $\delta$-phase reduces hence to finding the spin direction in the sheets as well as the stacking sequence of the sheets along $\langle 001\rangle$. The apparent absence of any magnetic signal at positions of $(00 l)$ reflections signals that spins are indeed aligned in the $(a-b)$ planes, or close to it, as expected. Pattern calculations and refinements show that the out-ofplane angle in LTC $\delta-\mathrm{O}_{2}$ cannot be larger than $\sim 25^{\circ}$, and is much smaller for the HTC phase. Since all these angles are close to their refined estimated standard deviation, and given the evidence from $\alpha-\mathrm{O}_{2}$, the $z$ component of the spins can be set to zero. With this setting, the orientation of the spins within the sheets is tightly constrained to be close to $\langle 010\rangle$. This is because the intensity of the reflections between $34^{\circ}$ and $44^{\circ}$ (Fig. 2) is extremely sensitive to the angle with respect to $\langle 010\rangle$, and their apparent absence (extreme weakness) is direct evidence of the spin alignment along $b$. To derive the stacking sequence of the sheets, the simplest conceivable scenarios correspond to either ferro- or antiferromagnetic coupling of neighboring sheets, i.e., $(A-A-A)$ or $(A-B-A)$ (Fig. 3). The first case produces the formerly proposed LTC structure, the latter the HTC structure. The excellent fits (Fig. 2) leave very little doubt on the correctness of these assignments. The assignment of the ITC structure is less obvious. The position of the peak at $2 \theta=26.5^{\circ}$ indicates $c=6.767 \AA$, i.e., doubling of the nuclear $c$-axis with respect to the LTC and HTC phases. This leaves four nonequivalent stacking pos- 


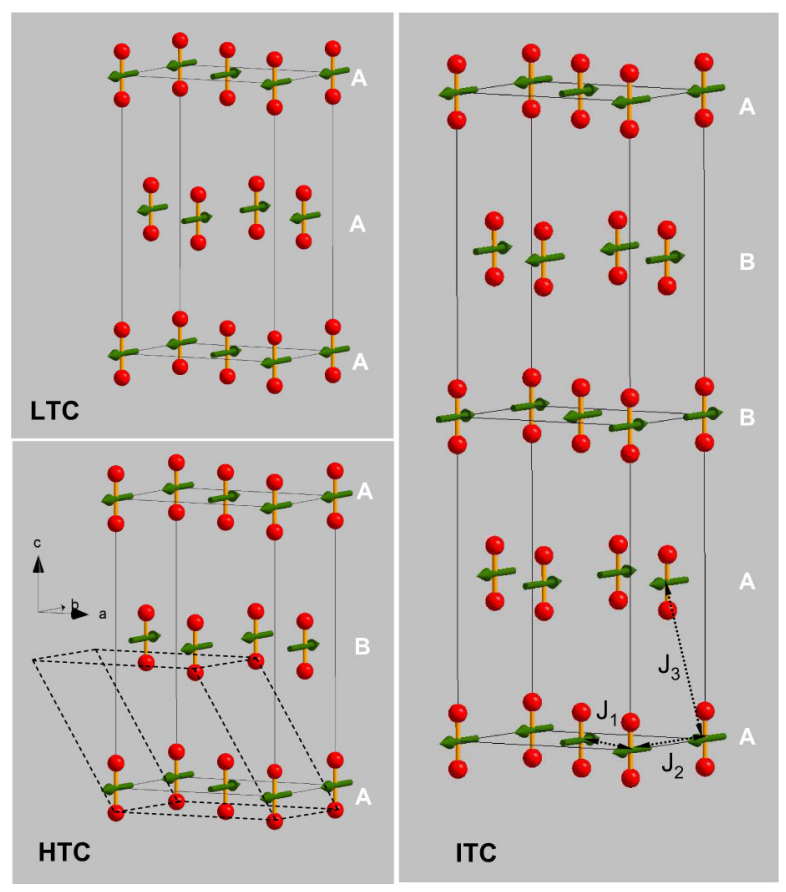

FIG. 3 (color online). Magnetic structures in $\delta-\mathrm{O}_{2}$. Upper Left: the low temperature commensurate (LTC) structure. Lower left: the high temperature commensurate (HTC) phase. Right: the intermediate commensurate (ITC) phase. Stability fields of the three phases are shown in Fig. 1. The unit cell of the low-pressure $\alpha$-phase (dashed) illustrates the close structural relationship between the two phases, having identical magnetic structures. " $A$ " and " $B$ " denote magnetic stacking sequence, $J_{1}$, $J_{2}$, and $J_{3}$ the intra- and interplane exchange parameters.

sibilities: $(A-B-B-B-A),(A-A-A-B-A),(A-A-B-A-A)$ and $(A-A-B-B-A)$. Pattern calculations reveal strongly different intensities for these configurations, which can be easily distinguished by the experiment. This eliminates all but one possibility, $(A-A-B-B-A)$, which corresponds to the fit in Fig. 2 and the ITC structure shown in Fig. 3. It is important to recall that the apparent lack or absence of magnetic Bragg peaks at large scattering angles does not arise from a rapid decrease of the magnetic form factor, but from the peculiar high-symmetric nature of all three magnetic structures which entails an almost or complete cancellation of most of the peaks up to $2 \theta \sim 45^{\circ}$. In all three cases we find a magnetic moment of magnitude $m=1.4 \pm$ $0.4 \mu_{B}$, which agrees within our uncertainty to the values reported from $\alpha$ - and $\beta-\mathrm{O}_{2}\left(1.64 \pm 0.06 \mu_{B}\right.$ and $1.55 \pm$ $0.03 \mu_{B}$, respectively) [9].

As mentioned further above, there is very little evidence of changes in nuclear structures across the two magnetic transitions, and this is corroborated by the refined lattice parameters of data collected along trajectory 1 (Fig. 1), as shown in Fig. 4. The magnetic transitions seem to have negligible influence on the nuclear structure. The data also demonstrate that the thermal expansion is negative in $c$ direction, and to a lesser extent also along $b$. The positive volume expansion arises hence solely from the strong positive thermal expansion along $a$. This is not unusual for solid oxygen: the $\alpha$ and $\beta$ phase both have negative thermal expansion along $b$ and $c$, respectively. This phenomenon is most likely caused by a strongly anisotropic thermal motion. Although the values of our anisotropic thermal displacement parameters are not very reliable and scatter across the 3 samples and $\sim 100$ patterns collected in the different loadings, the refined thermal motion along the $a$ axis is systematically $\sim 3$ times larger than along $c$. In other words, the $\mathrm{O}_{2}$ molecule librates, possibly with a preference around its spin direction (thereby avoiding canting), which naturally leads to a positive and negative thermal expansion along $a$ and $c$, respectively.

To resume, the magnetic phase diagram of $\delta$-oxygen contains three different phases and is hence considerably richer than has been presumed so far. Although we did not measure above $235 \mathrm{~K}$, magnetic order is likely to exist up to room temperature given the spectroscopic evidence [3] and the fact that there is no detectable change of the magnetic moment over a temperature range of $200 \mathrm{~K}$.

We believe that the key to the understanding of these unusual transition sequences is the very strong dependence of the $p$-electron based exchange coupling on the orientation of $\mathrm{O}_{2}$ molecules in neighboring sheets. It is abundantly clear that the magnetic phase diagram of solid oxygen can be described by a Heisenberg Hamiltonian which includes three coupling constants: $J_{1}$ (intraplane, between parallel spins), $J_{2}$ (intraplane, between antiparallel spins), and $J_{3}$ (interplane, nearest neighbors). Values for $\delta-\mathrm{O}_{2}$ are unknown, but have been derived for the closely related $\alpha$-phase from both measurements and calculations [2]. Whereas $J_{1}$ and $J_{2}$ are large (58 and 14-28 K, respectively), reported values for $J_{3}$ are very small in magnitude $(<1 \mathrm{~K})$, and either positive or negative, depending on the reference [2]. Van Hemert et al. [10] investigated the orientation and distance dependence of coupling constants in an $\mathrm{O}_{2}-\mathrm{O}_{2}$ dimer, and find $J_{3}$ positive (ferromagnetic) hence consistent with the experimental finding in LTC $\delta-\mathrm{O}_{2}$, and close to the point where it changes sign. The important point here is that $J_{3}$ is in fact negative (antiferromagnetic coupling) for most of the mutual orientations, except the "parallel shifted" orientation found in $\delta$ - and $\alpha-\mathrm{O}_{2}$ between nearest neighbor molecules in adjacent sheets (see Fig. 3). The thermal motion (libration) leads to sizeable temporal distortions from such orientations, as already pointed out by Etters et al. [11] who find fluctuations of the molecular axis by $\approx 19^{\circ}$, thereby approaching either the "T" (molecular axes perpendicular) or "parallel" (molecular axes parallel) orientations that both have strong antiferromagnetic coupling [10]. In other words, it is evident that the librational motion has a critical influence on the sign of the effective (i.e., motional averaged) magnetic exchange coupling constants, and in particular $J_{3}$ which governs the magnetic stacking sequence in the $c$ direction. If this is correct, the LTC-ITC-HTC transitions should approximately follow lines of constant librational fluctuations $\left\langle u^{2}\right\rangle$. To evaluate this we use the relation 


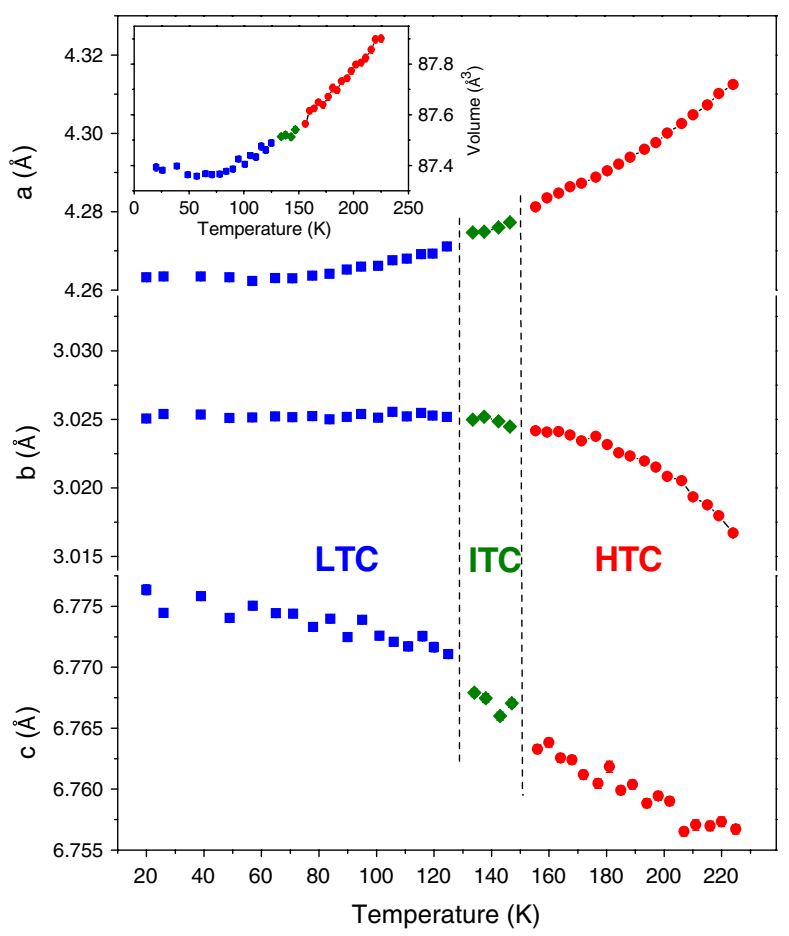

FIG. 4 (color online). Temperature dependence of the lattice parameters (main figure) and nuclear unit cell volume (inset) along the trajectory 1 shown in Fig. 1 . Note that the path is not strictly isobaric

$$
(\partial T / \partial P)_{\left\langle u^{2}\right\rangle}=-\frac{\left(\partial \ln \left\langle u^{2}\right\rangle / \partial P\right)_{T}}{\left(\partial \ln \left\langle u^{2}\right\rangle / \partial T\right)_{P}} .
$$

From the refined thermal parameters along trajectory 1 (Fig. 1) we obtain $\left(\partial \ln \left\langle u^{2}\right\rangle / \partial T\right)_{P}=5 \times 10^{-3} \mathrm{~K}^{-1}$. For the quantity in the numerator we use an Einstein model $\left\langle u^{2}\right\rangle=(3 h / 2 m \nu)$ with an average libron frequency of $\nu=70 \mathrm{~cm}^{-1}$ as determined from optical measurements [2]. This gives $\left(\partial \ln \left\langle u^{2}\right\rangle / \partial P\right)_{T}=-(\partial \ln \nu / \partial P)_{T}=$ $-8 \times 10^{-2} \mathrm{GPa}^{-1}$, taking again the high pressure optical data [2], and hence $(\partial T / \partial P)_{\left\langle u^{2}\right\rangle}=16 \mathrm{~K} / \mathrm{GPa}$. This value is in excellent agreement with the measured value of $\approx 15 \mathrm{~K} / \mathrm{GPa}$ as shown in Fig. 1. Thus, the value of the slope of the LTC-ITC-HTC transition lines supports the conjecture that the influence of the thermal motion on the exchange parameter $J_{3}$ is responsible for the peculiar magnetic transitions in $\delta-\mathrm{O}_{2}$.

The fact that within the same nuclear structure three different magnetic structures exist casts some doubt on the notion of solid oxygen being "spin-controlled" [5], i.e., the belief that the phase diagram is completely imposed by the magnetic structures. There is no question that the inplane magnetic interactions have a major effect on the corresponding nuclear parameters as seen by the distortion of the $a-b$ plane from strictly hexagonal (as found in $\beta-\mathrm{O}_{2}$ ) to orthorhombic with $a / b \approx 1.41\left(\alpha\right.$ - and $\delta$ - $\left.\mathrm{O}_{2}\right)$ compared to $\sqrt{3}=1.73$. However, the responsible interactions $\left(J_{1}\right.$, $J_{2}$ ) are strong, which is not the case for the interplane interaction $\left(J_{3}\right)$ which determines the stacking sequence of the $\mathrm{O}_{2}$ sheets. The fact that HTC $\delta-\mathrm{O}_{2}$ and $\alpha-\mathrm{O}_{2}$ have strictly identical magnetic structures at the same temperatures and similar volumes (there is no detectable volume change at the $\alpha-\delta$ transition [2]) suggests that the notion of solid oxygen being "spin controlled" is either overstretched or that there are yet unrecognized subtle differences in the magnetic structures of the two phases.

We conclude with the remark that the apparent disappearance of magnetic order at $\sim 150 \mathrm{~K}$ reported in Ref. [4] is almost certainly due to a gradual pressure increase during heating of the clamped pressure cell used in this study, as already suspected in Ref. [3]. As the sample pressure approached the $\delta$ - $\epsilon$ transition line the magnetic reflections disappeared since $\epsilon-\mathrm{O}_{2}$ is nonmagnetic.

We thank J.L. Laborier, C. Payre (ILL), G. Hamel (IMPMC), and C. L. Bull (CSEC) for technical assistance, as well as the DOENNSA Cooperative agreement DEFC52-06NA26274 and the Université P\&M Curie in Paris for financial support during the sabbatical stay of A. L. C. in 2009 at the IMPMC.

*Stefan.Klotz@impmc.jussieu.fr

[1] D. A. Young, Phase Diagrams of the Elements (University of California Press, Berkeley, 1991).

[2] Y. A. Freiman and H. J. Jodl, Phys. Rep. 401, 1 (2004).

[3] F. Gorelli, M. Santoro, R. Bini, and L. Ulivi, Phys. Rev. B 77, 132103 (2008).

[4] I. N. Goncharenko, Phys. Rev. Lett. 94, 205701 (2005).

[5] I. N. Goncharenko, O.L. Makarova, and L. Ulivi, Phys. Rev. Lett. 93, 055502 (2004).

[6] All pressure values cited here were determined from the measured (refined) unit cell volume and an equation of state based at high temperatures on the $300 \mathrm{~K}$ data of Schiferl et al. (Ref. [7]) and at low temperatures the $19 \mathrm{~K}$ data from Akahama et al. (Ref. [8]), assuming an average thermal expansion of $8 \times 10^{-5} \mathrm{~K}^{-1}$, as well as no volume difference between $\alpha$-, $\beta$-, and $\delta$-O $\mathrm{O}_{2}$ (Ref. [2]). This constrains the error on pressure values to be less than $\pm 0.15 \mathrm{GPa}$.

[7] D. Schiferl, D. T. Cromer, and R. L. Mills, Acta Crystallogr. Sect. B 39, 153 (1983).

[8] Y. Akahama, H. Kawamura, and O. Shimomura, Phys. Rev. B 64, 054105 (2001).

[9] R. J. Meier and R. B. Helmholdt, Phys. Rev. B 29, 1387 (1984).

[10] M. C. V. Hemert, P.E. S. Wormer, and A. V. der Avoird, Phys. Rev. Lett. 51, 1167 (1983).

[11] R. D. Etters, A. A. Helmy, and K. Kobashi, Phys. Rev. B 28, 2166 (1983).

[12] T. C. Hansen, P. F. Henry, H. E. Fischer, J. Torregrossa, and P. Convert, Meas. Sci. Technol. 19, 034001 (2008).

[13] T. Strässle, S. Klotz, G. Hamel, M. M. Koza, and H. Schober, Phys. Rev. Lett. 99, 175501 (2007).

[14] J. Rodriguez-Carvajal, Physica (Amsterdam) 192B, 55 (1993). 